\title{
ANÁlISIS DEl SISTEMA DE ARRANQUE EN NEGRO EN LA REPÚbliCA DOMINICANA
}

\author{
Analysis of the black-start system in the Dominican Republic
}

\author{
Freddy Lázaro Acosta Riveraa , Fanuel Antonio Ferrer Rivas ${ }^{\mathrm{b}}$, \\ Jean Carlos Milanés Rodríguez ${ }^{c}$, Doris Alexandra Nina de la \\ Cruz ${ }^{\text {d }}$, Ransys Antonio Santos Tejeda ${ }^{e}$, Eduardo Agustín de \\ León Concepción ${ }^{\mathrm{f}}$ y Miguel Euclides Aybar Mejíag
}

Recibido: $19 / 07 / 19 \bullet$ Aprobado: 16/12/19

Cómo citar: Acosta Rivera, F. L., Ferrer Rivas, F. A., Milanés Rodríguez, J. C., Nina de la Cruz, D. A., Santos Tejeda, R. A., de León Concepción, E. A., \& Aybar Mejía, M. E. (2019). Análisis del sistema de arranque en negro en la República Dominicana. Ciencia, Ingenierías y Aplicaciones, 2(2), 29-49. Doi: https://doi. org/10.22206/cyap.2019.v2i2.pp29-49

\section{Resumen}

República Dominicana es un país con un sistema eléctrico vulnerable, por ello las fallas son recurrentes y las pérdidas parciales y totales del sistema son una consecuencia latente en las últimas décadas, siendo el tiempo de restablecimiento el factor de mayor importancia en estos eventos. Por otro lado, la República Dominicana se encuentra en la trayectoria de los huracanes, por lo que la red eléctrica del país se ve amenazada en la temporada ciclónica. Ante esta situación, este pais no cuenta con un esquema que permita restablecer el sistema en caso de que algún fenómeno o evento natural ocasione una falla en el suministro eléctrico. En el presente documento se plantea un método de remuneración y un esquema para el restablecimiento del sistema de forma rápida y eficaz; esto se logra conociendo los puntos vulnerables del sistema, las generadoras con la capacidad de realizar el servicio de blackstart y con la ayuda de herramientas de simulación para estimar el tiempo de respuesta del sistema. Para la realización de este programa se plantea la

\footnotetext{
a Ingeniería Eléctrica, Instituto Tecnológico de Santo Domingo (INTEC), República Dominicana Correo-e: freddylazaro08@gmail.com

b Correo-e: fanuel0214@gmail.com

${ }^{c}$ Correo-e: jmilanesrodriguez13@gmail.com

d Correo-e: dorisnina06@gmail.com

e Correo-e: rast.21@gmail.com

${ }^{\mathrm{f}}$ Correo-e: eduardo.deleon@intec.edu.do

g Correo-e: miguel.aybar@intec.edu.do
} 
situación actual del sistema y se compara con paises similares que tengan un sistema de restablecimiento óptimo, analizando las maniobras y variables tomadas en cuenta para su realización. Adicionalmente, se plantea el impacto de este algoritmo en los ámbitos económico, social, legal, técnico y ambiental evidenciando el antes y después del sistema eléctrico dominicano con la implementación de la propuesta, además de observarse los ahorros financieros que tendría el Sistema Eléctrico Nacional Interconectado (SENI) al implementar dicha metodología.

Palabras clave: blackout; black-start; despacho; maniobra; restablecimiento; DIgSILENT

\begin{abstract}
The Dominican Republic is a country with a vulnerable electrical system. Failures in this are recurrent and partial and total losses of the system have been a latent consequence in the last decades, being the time of restoration, the most important factor in these events. The Dominican Republic is in the path of hurricanes, so the country's electricity grid is threatened in hurricane season, given this situation, there is no scheme to restore the system when these phenomena cause a blackout in the country. In this document we propose a method of remuneration and a scheme for the restoration of the system quickly and efficiently. This is achieved by knowing the vulnerable points of the system, the generators with the ability to perform the black-start service and with the help of simulation tools to estimate the response time of the system. To carry out this program, the current situation of the system is considered and compared with similar countries that have an optimal restoration system analyzing the maneuvers and variables considered for their realization. In addition, the impact of this algorithm is raised in the economic, social, legal, technical and environmental areas, evidencing the before and after of the Dominican electricity system with the implementation of the proposal, in addition to observing the financial savings that the National Interconnected Electric System (SENI) would have when implementing this methodology.
\end{abstract}

Keywords: Blackout; black-start; dispatch; maneuver; restoration; DIgSILENT. 


\section{Introducción}

Las fallas eléctricas (también conocidas como interrupciones en cascada o blackouts) causan grandes pérdidas financieras y traen desabastecimiento de electricidad a los usuarios de la red eléctrica (Wu, Chang, \& Hu, 2017). Los blackouts son eventos costosos que amenazan la integridad de los sistemas de energía eléctrica en todo el mundo (Kamali \& Amraee, 2017), por lo tanto, las vulnerabilidades de tales instalaciones deben minimizarse para hacer frente a varias fuentes de interrupción en los sistemas de energía eléctrica (Abedi, Gaudard, \& Romerio, 2019).

El sistema eléctrico de potencia se puede definir como el conjunto de una red que contiene generadores de energía eléctrica, transformadores de voltaje, sistemas de protección, cables de alimentación de conexión y dispositivos de recepción de energía. (Masnicki \& Mindykowski, 2017). En la República Dominicana el sistema eléctrico tiene una capacidad de potencia instalada de 3,702.81 MW. De este total, los motores de combustión interna y turbinas de ciclo combinado alcanzan el $65.3 \%$ de la generación, siendo así los principales responsables de sostener el sistema. Esto podría variar de cara al futuro, con la creación de plantas generadoras con nuevas tecnologías, distintas a las antes mencionadas (Organismo Coordinador, 2018).

Dicho sistema tiene una larga historia de colapsos; al respecto, se pueden enumerar alrededor de 85 blackouts desde el 1988 hasta la fecha, algunos de ellos alcanzan periodos superiores a las 48 horas, donde un $54.12 \%$ de estos ocurrieron en temporadas ciclónicas, dando a entender que el sistema de potencia dominicano se hace vulnerable al paso de los huracanes (Centro de Control de Energía, 2018). A pesar de la excesiva cantidad de blackouts, larga duración de estos, y su vulnerabilidad ante huracanes, aún no se cuenta con una metodología que dé los pasos a seguir ante la ocurrencia de una situación de este tipo.

Esta investigación tiene como objetivo principal elaborar un algoritmo que sirva de guía y reduzca el tiempo de restablecimiento del sistema 
eléctrico frente a una pérdida total del suministro eléctrico. Además, se determinarán las plantas con capacidad de arrancar en negro y la remuneración que recibirían las plantas que ofrezcan dichos servicios.

\section{Estudio de la situación actual del arranque en negro en República Dominicana}

\section{Esquema de restablecimiento actual}

Las estrategias para la restauración de blackout dependen en gran medida de la ubicación, de las características de las unidades de blackstart, la activación de la línea, la recuperación de carga, la formación de islas y la sincronización del sistema se consideran factores importantes (Kurup \& Ashok, 2015).

El esquema de restablecimiento del Sistema Eléctrico Nacional Interconectado (SENI) no es más que la secuencia de maniobras a seguir para poner en marcha la red luego de un apagón total o parcial de la misma. Es imprescindible para cualquier sistema de potencia contar con esta contingencia, ya que de este dependerán la seguridad y confiabilidad que se pueda brindar a los usuarios. Según la información obtenida en diferentes entrevistas a agentes del sector, se determinó que anteriormente no existía un esquema oficial de restablecimiento del sistema eléctrico. Tampoco existía un procedimiento formal complementario que mencionara los criterios técnicos, económicos y regulatorios que se deben seguir para su utilización de este servicio. El restablecimiento del SENI se realizaba de manera empírica donde el punto de partida eran las unidades de generación hidroeléctrica, ya que estas son capaces de brindar servicio de arranque autógeno y se encuentran habilitadas para esto.

\section{Pérdidas por blackout}

Los blackout a gran escala ocurren raras veces en los sistemas de potencia, pero cuando estos suceden provocan enormes pérdidas económicas y sociales (Yusuf, Sakib, \& Ahsan, 2016). 
El 16 de mayo del 2015, en el período 23, ocurrió una salida total del Sistema Eléctrico Nacional Interconectado (SENI) fruto de un desprendimiento de uno de los contactos del seccionador de barra, provocando una falla monofásica en la fase B de ITABO GAS (Organismo Coordinador, 2015). La gráfica 1, que se presenta a continuación, muestra el comportamiento de la potencia en el SENI al momento del evento, siendo AES Andrés la unidad que entregaba mayor potencia, $278 \mathrm{MW}$, seguida por Quisqueya 2 con 185.7 MW. Ambas son centrales de generación térmica, una de ciclo combinado y otra con motores de combustión interna, las cuales por su tecnología pueden ser consideradas para el restablecimiento del SENI.

Período 23 del 16-05-2015

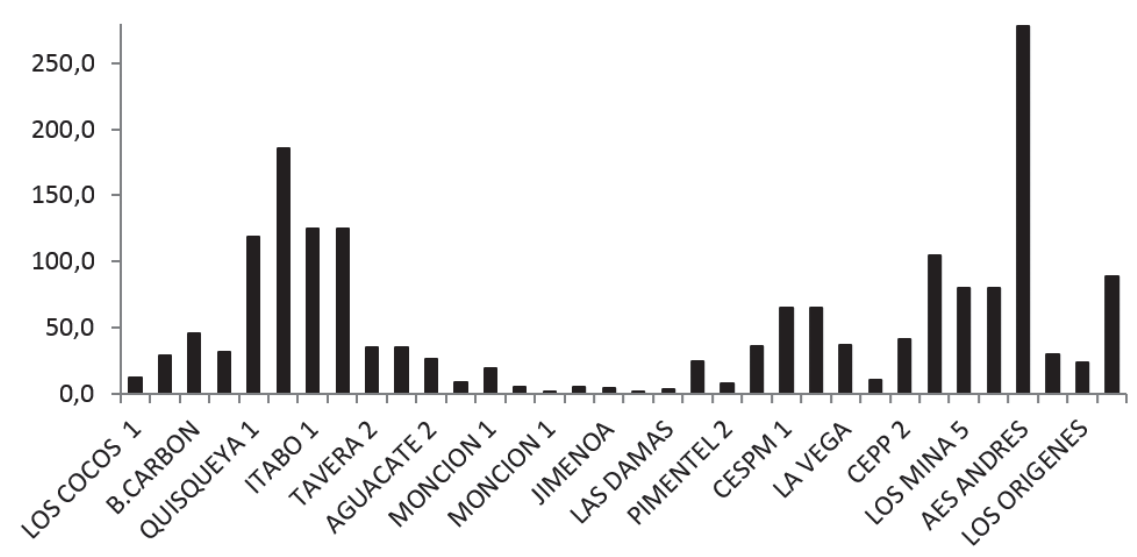

Figura 1. Potencia suministrada por los generadores previo al apagón del 2015

En la gráfica 2 se evidencia el comportamiento de la demanda abastecida real contra la generación programada, para los períodos en que se interrumpió el servicio por motivos de la falla. En dicha gráfica se representa el déficit energético o energía no suministrada a los consumidores finales. Se puede observar que el área bajo la curva de desabastecimiento representa la masa total de energía no entregada y, por ende, pérdida del sistema que asciende a un total de $22.4 \mathrm{GWh}$. 


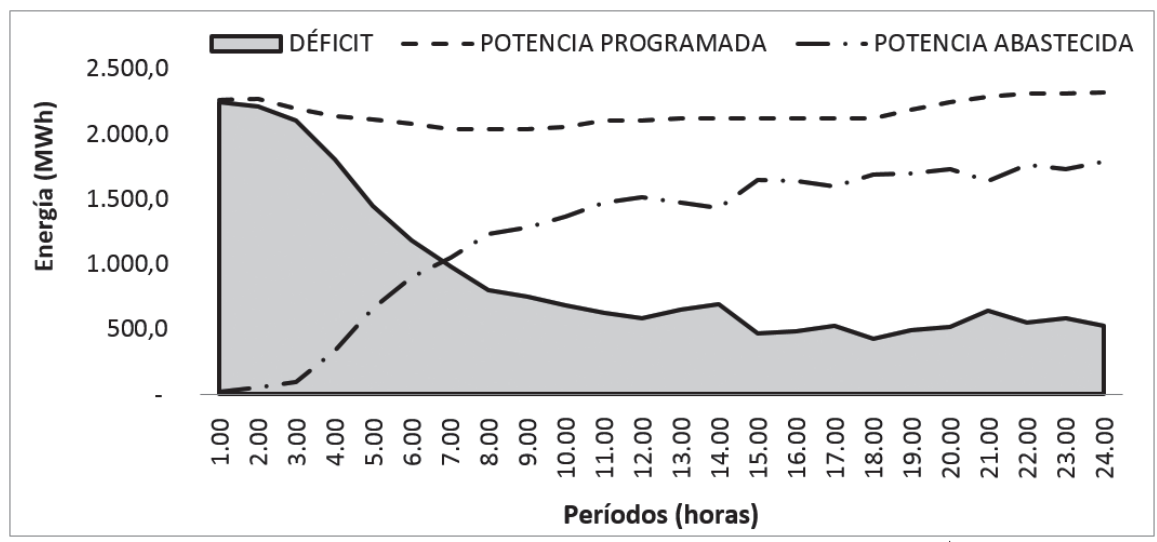

Figura 2. Evolución del abastecimiento durante el blackout 16/17-05-2015

Normalmente, para 24 períodos en el SENI, para el 2015, por gestión de demanda y déficit de generación se dejaban de abastecer unos 7.5 GWh. Al comparar esta magnitud con los $22.4 \mathrm{GWh}$ del análisis del evento, se puede observar como este valor es casi tres veces mayor que el desabastecimiento convencional, sin mencionar que las causas del blackout probablemente afectan también a la infraestructura de la red, y otras pérdidas que no se cuantifican en balances de energía.

Esta energía no suministrada le cuesta al sistema el máximo precio al que podría valorizarse en las transacciones: costo de desabastecimiento, ya que frente a este priman costos superiores a los convencionales de producción y despacho. Se hace evidente, entonces, la magnitud del impacto de un blackout en el Sistema Eléctrico Nacional Interconectado de la República Dominicana, desde el punto de vista energético.

\section{Unidades con la capacidad de arranque en negro}

La restauración del sistema de energía después de un apagón de gran área se conoce como Black Start (Leng, Lu \& Liang, 2018). Esta tarea es una tarea crítica para reducir las pérdidas económicas y el malestar social causado por el apagón, que se utiliza para reestablecer el servicio eléctrico después de un apagón general (Yang, Liu \& Liu, 2014). 
En el sistema eléctrico nacional de la República Dominicana se utilizan las centrales hidroeléctricas como centrales para restablecer el sistema en la ocurrencia de un blackout. Existen centrales generadoras que son capaces de brindar el servicio de black-start y otras que pueden ser adecuadas para brindar dicho servicio. Las unidades generadoras que pueden realizar el arranque en negro deben cumplir varios requisitos para realizar la maniobra, esto depende del tipo de tecnología de la unidad, entre estos se encuentran:

- Poder arrancar con barra muerta.

- Tener la frecuencia dentro de los parámetros establecidos.

- Contar con reserva como mínimo por cinco horas.

- Tener sistemas de telecomunicaciones.

Actualmente, en el país solo algunas unidades hidráulicas pueden realizar el servicio de arranque en negro, debido a que la cantidad de centrales con este servicio debe aumentar para así disminuir el tiempo de restablecimiento. A continuación, se presenta el gráfico 3 con un mapa de la ubicación de cada una de las centrales que están realizando el servicio, y aquellas que respondieron al organismo coordinador que pueden realizarlo o que necesitan modificaciones, ubicadas en la zona Norte, la zona Central-Sur y la zona Este.

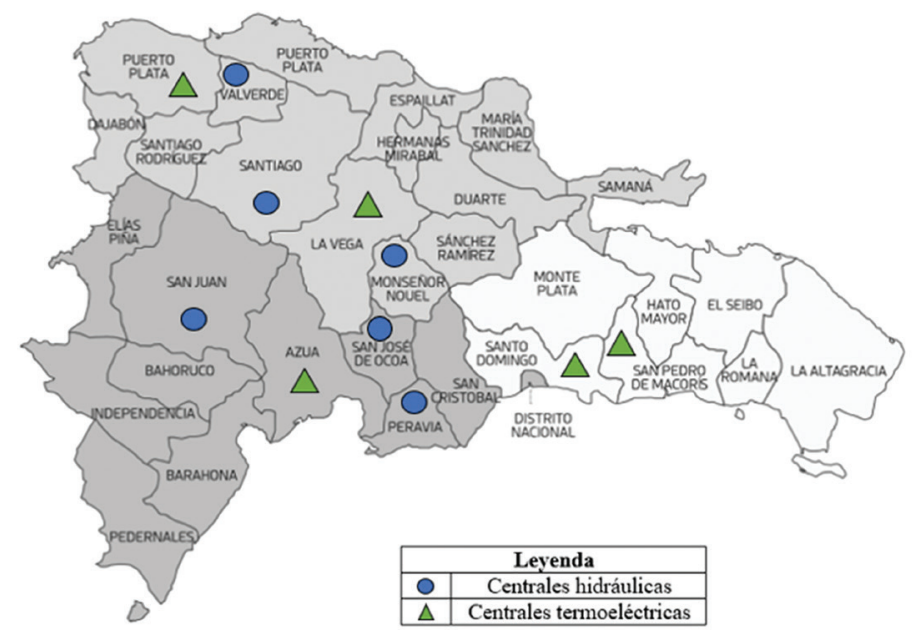

Figura 3. Ubicación geográfica de las centrales hidráulicas y termoeléctricas con la capacidad de realizar el arranque en negro. 
En las tablas 1 y 2 se muestran las especificaciones más relevantes de estas unidades mencionadas, además están divididas dependiendo de la tecnología de generación que estas usen, la tabla 1 presenta las centrales hidráulicas y la tabla 2 las centrales termoeléctricas.

Tabla 1. Especificaciones técnicas de las unidades hidroeléctricas con capacidad para el servicio del arranque en negro

\begin{tabular}{ccccc}
\hline Ítem & Central eléctrica & $\begin{array}{c}\text { Potencia } \\
\text { Nominal } \\
(\mathbf{M W})\end{array}$ & Ubicación & Propietario \\
\hline 1 & Jiguey & 49 & Peravia & \\
2 & Tavera & 48 & Santiago & \\
3 & Palomino & 40.8 & San Juan & \\
4 & Aguacate & 30 & Peravia & Empresa de \\
5 & Valdesia & 27 & Peravia & Generación \\
6 & Monción & 26 & Valverde & Hidroeléctrica \\
7 & Pinalito & 25 & Monseñor Nouel & \\
8 & Río Blanco & 12.5 & Monseñor Nouel & \\
9 & Sabaneta & 6.3 & San Juan &
\end{tabular}

Tabla 2. Especificaciones técnicas de las unidades termoeléctricas con capacidad para el servicio del arranque en negro

\begin{tabular}{ccccccc}
\hline Ítem & Central & Tecnología & $\begin{array}{c}\text { Potencia } \\
\text { Nominal } \\
(\mathbf{M W})\end{array}$ & $\begin{array}{c}\text { Mínimo } \\
\text { Técnico } \\
(\mathbf{M W})\end{array}$ & Ubicación & Propietario \\
\hline 1 & San Felipe & Turbina Gas & 185.00 & 132 & Puerto Plata & San Felipe \\
2 & Quisqueya 1 & Motor Diesel & 150.00 & 66 & $\begin{array}{c}\text { San Pedro de } \\
\text { Macorís }\end{array}$ & PVDC \\
3 & Palamara & Motor Diesel & 93.24 & 21.2 & Santo Domingo & GPLV \\
4 & Monte Rio & Motor Diesel & 93.20 & 45 & Azua & Monte Rio \\
5 & La Vega & Motor Diesel & 51.00 & 1.85 & La Vega & GPLV \\
6 & CEPP 2 & Motor Diesel & 31.20 & 11 & Puerto Plata & CEPP \\
7 & CEPP 1 & Motor Diesel & 10.40 & 4.48 & Puerto Plata & CEPP
\end{tabular}




\section{Propuesta para el restablecimiento en República Dominicana}

\section{Lista de orden de entrada de las centrales con capacidad de blackstart}

El orden de mérito describe en qué orden de entrada se utilizan las fuentes de energía que contiene una matriz energética de un país. Según los costos marginales, se clasifican en tecnologías de bajo costo de producción de energía hasta la más cara de la siguiente manera: energías renovables intermitentes (fotovoltaica y eólica), hidroeléctrica, nuclear, carbón, gas, petróleo (Janda, 2018). Con esto los coordinadores del sector eléctrico proporcionan a los consumidores la electricidad más barata posible.

Para la determinación del orden de entrada de las centrales, no existirá un lineamiento rígido a seguir cada vez que sea necesario el restablecimiento del sistema. La operación es flexible y las contingencias son inesperadas. Se analizarán las unidades en función de sus características de potencia y tiempo. Potencia, porque este parámetro determina el peso que tiene la unidad en el restablecimiento, mientras que el tiempo de arranque se traduce a una reducción del tiempo total que tarda el esquema de maniobras en ser ejecutado.

La metodología de restablecimiento debe ser desarrollada por zonas o islas. Por lo tanto, con base en la ubicación de las unidades hidroeléctricas de mayor potencia se establecerán rutas o zonas convenientes. En la actualidad, en la República Dominicana las hidroeléctricas declaran una capacidad específica de generación inferior a la nominal, de forma que se garantice el óptimo aprovechamiento de los recursos hídricos. En cuanto a las unidades termoeléctricas que se consideran para este estudio, se tomó de referencia la comunicación del Organismo Coordinador OC-2018000845, dando respuesta al comunicado SIE-E-CSIE-SI-2018-0438 donde se convoca a los agentes del Mercado Eléctrico Mayorista (MEM) a una reunión para deliberar cuáles unidades del SENI podrían habilitarse para realizar el arranque en negro. Aunque estas centrales requieran adecuaciones, se debe evaluar la posibilidad de que con el sistema de remuneración las empresas generadoras podrán incurrir en estos costos y brindar el servicio. Se agruparán los generadores por zona operativa, y 
luego se determinará el orden con que deberán entrar en línea. En la tabla 3 se muestran las unidades por zona en el orden que se propone para la entrada de las centrales. Se evidencia que no existen unidades habilitadas para realizar el arranque en negro en la zona este.

Tabla 3. Lista de orden de entrada de unidades con capacidad de arranque en negro por zona

\begin{tabular}{|c|c|c|c|c|}
\hline $\begin{array}{c}\text { Zona } \\
\text { Operativa }\end{array}$ & Centrales & Tecnología & $\begin{array}{l}\text { Capacidad } \\
\text { (MW) }\end{array}$ & $\begin{array}{l}\text { Tiempo de } \\
\text { arranque } \\
\text { (h) }\end{array}$ \\
\hline \multirow{10}{*}{ Sur-Central } & JIGUEY 1 & Hidro & 49 & - \\
\hline & JIGUEY 2 & Hidro & 49 & - \\
\hline & PALOMINO 1 & Hidro & 40.8 & - \\
\hline & PALOMINO 2 & Hidro & 40.8 & - \\
\hline & VALDESIA 1 & Hidro & 27 & - \\
\hline & VALDESIA 2 & Hidro & 27 & - \\
\hline & PINALITO 1 & Hidro & 25 & - \\
\hline & PINALITO 2 & Hidro & 25 & - \\
\hline & PALAMARA & Diesel & 93.24 & 0.13 \\
\hline & MONTE RIO & Diesel & 93.2 & 0.25 \\
\hline \multirow{12}{*}{ Norte } & TAVERA 1 & Hidro & 48 & - \\
\hline & TAVERA 2 & Hidro & 48 & - \\
\hline & MONCIÓN 1 & Hidro & 26 & - \\
\hline & MONCIÓN 2 & Hidro & 26 & - \\
\hline & RÍO BLANCO 1 & Hidro & 12.5 & - \\
\hline & RÍO BLANCO 2 & Hidro & 12.5 & - \\
\hline & SABANETA & Hidro & 6.3 & - \\
\hline & CEPP 1 & Diesel & 10.4 & 0.28 \\
\hline & QUISQUEYA 1 & Diesel & 150 & 0.5 \\
\hline & LA VEGA & Diesel & 51 & 0.95 \\
\hline & CEPP 2 & Diesel & 31.2 & 2 \\
\hline & SAN FELIPE & T. Gas & 185 & 6 \\
\hline
\end{tabular}

La estrategia planteada para el restablecimiento es tipo build-up, es decir, se va levantando el sistema mediante la formación de islas que 
contengan por lo menos una central con capacidad de arranque en negro y una unidad termoeléctrica a vapor (Hou, Liu, Zhang \& Sun, 2009). En dicha estrategia se les da prioridad a las centrales hidroeléctricas debido a su reducido tiempo de arranque frente a las demás tecnologías. De igual manera, de no estar disponible la primera unidad de la lista se asume la siguiente para iniciar la maniobra, y así sucesivamente.

\section{Tiempo restablecimiento zona central}

El rendimiento y desviación estándar de las rutas establecidas serán calculados de acuerdo a (Mota, Mota \& Morelato, 2007), tomando el tiempo pésimo, el tiempo más probable y el tiempo óptimo en que se puede realizar cada actividad de la secuencia que se sigue paso a paso para el restablecimiento. El rendimiento y la desviación estándar están dados por las expresiones:

$$
\begin{aligned}
& t_{e}=\frac{t_{o}+4 t_{m}+t_{p}}{6} \\
& \sigma_{t}=\frac{t_{p}-t_{0}}{6}
\end{aligned}
$$

Donde,

$\mathrm{t}_{\mathrm{e}}=$ rendimiento

$\mathrm{t}_{0}=$ tiempo óptimo

$\mathrm{t}_{\mathrm{m}}=$ tiempo más probable

$\mathrm{t}_{\mathrm{p}}=$ tiempo más pésimo

$\sigma_{\mathrm{t}}=$ desviación estándar

Se contemplan a su vez un tiempo de rendimiento y dos escenarios, uno más crítico y otro óptimo. El seguimiento de las maniobras determinará el tiempo que tardará el restablecimiento. Se analizarán los tiempos con el esquema mostrado y luego, el tiempo, ańadiendo los motores diesel y turbinas de gas que pueden a su vez realizar un arranque instantáneo, y potencialmente reducir aún más dicho tiempo. 


\section{Sistema de remuneración propuesto}

El objetivo de remunerar este servicio complementario en República Dominicana es conseguir la disposición de la mayor cantidad de generadoras para poder brindar el servicio en caso de un colapso total de SENI. Asimismo, mientras mayor es la cantidad de unidades disponibles el tiempo de restablecimiento es mucho menor, creando un sistema eléctrico con mucho más confiabilidad y seguridad.

Para la condición actual de la República Dominicana se escogió el esquema de contrato bilateral, debido a que en este mercado existen contratos que involucran relaciones comerciales entre los agentes productores de energía y los usuarios consumidores. En República Dominicana, los consumidores, a través de un comercializador (en este caso las Empresas de Distribución Eléctrica), adquieren el suministro de energía por parte de los generadores.

El contrato bilateral que se propone solo tiene en cuenta la remuneración por disponibilidad en blackstart en caso de que surja un colapso, la energía suministrada en ese momento será pagada al precio de reconciliación. En el siguiente apartado se muestra la Ec. 3 con la manera de calcular dicho precio:

$$
\operatorname{PR}(\$ / M W h)=\operatorname{Max}[\text { PrecioBolsa,POferta }](\text { Ec. 3) }
$$

Donde:

$\operatorname{PR}(\$ / M W h)=$ Precio de reconciliación aplicado a la unidad generadora que suministró energía en momentos de colapso.

PrecioBolsa $(\$ / M W h)=$ Precio de la bolsa establecido por el mercado mayorista de electricidad en condiciones de operación normal del SENI.

POferta $(\$ / M W h)$ = Último precio de oferta entregado por el agente generador al mercado mayorista de electricidad. 
Por lo tanto, la remuneración por este servicio se determina como la Ec. 4:

$$
\operatorname{RENBS}_{\mathrm{ijt}}(\$)=\mathrm{ENBS}_{\mathrm{ijt}} * \text { PR (Ec. 4) }
$$

Donde:

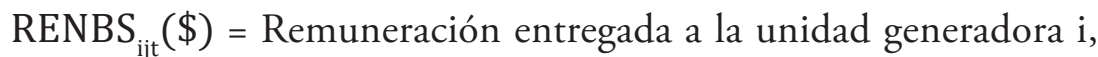
en el área operativa $\mathrm{j}$, debido a las obligaciones adquiridas en el periodo de contratación t.

$\mathrm{ENB}_{\mathrm{ijt}}(\mathrm{MWh})=$ Cantidad de energía utilizada de la planta con capacidad de autoarranque $i$, la cual pertenece al área operativa $j$, en el intervalo comercial t. Este valor es calculado por el operador del sistema.

$\operatorname{PR}(\$ / M W h)=$ Precio de reconciliación aplicado a la unidad generadora que suministró energía en momentos de colapso.

Otros factores importantes en la remuneración son:

- Penalizaciones. Al ser un servicio solicitado con baja periodicidad, se puede incurrir en el aprovechamiento de los incentivos sin brindar un mantenimiento efectivo a las unidades generadoras, por dicha razón se propone penalizar a las plantas por:

- No brindar el servicio al instante de ser requerido, con el pago total del servicio de la unidad que lo brindó cuando a esta le correspondía. Además de ser suspendido por tiempo indefinido sin remuneración alguna en el momento del incumplimiento.

- Presentar anomalías al momento de una prueba de retina para comprobar su estado, suspendiéndose el pago hasta que demuestre estar en las condiciones necesarias para proporcionar el servicio cuando sea requerido.

- Periodicidad. Tomando en consideración la modalidad del sistema, se propone que en el contrato se realicen pagos mensuales por el cargo de disponibilidad y junto con esto, anualmente, antes 
de concluir el contrato, se realice un pago por amortización de inversión debido a:

- Las transacciones del Mercado Eléctrico Mayorista (MEM) se realizan en períodos de corto y largo plazo. Sin embargo, la valorización de la operación de las centrales generadoras es reconocida de forma mensual.

- Repartición. Una salida total del sistema afecta a todos los agentes y usuarios, indistintamente. Por lo tanto, se deciden repartir los costos entre todo ente involucrado al SENI, en este caso, entre los usuarios y las unidades que no pueden realizar el servicio, con un $50 \%$, respectivamente. En el siguiente apartado se muestran los cálculos para determinar dicho valor. En el caso del $50 \%$ correspondiente a los usuarios este se dividirá entre todos los usuarios y se agregará a la factura de cada usuario regulado.

\section{Análisis de simulaciones}

El software utilizado para el desarrollo de las simulaciones es DIgSILENT Power Factory 2018, los parámetros de los elementos que conforman al SENI, fueron brindados por el Organismo Coordinador para la modelación de estos, además de hojas de cálculos digitales.

El plan de restauración describe los pasos que los operadores de transmisión deben seguir para restaurar el sistema fallado con ayuda de las unidades de blackstart. Esto incluye la energización secuencial de transformadores, líneas de transmisión, compensadores de reactivos y carga disponible, con el objetivo de suministrar energía a las cargas auxiliares en las plantas de destino, seleccionadas para el restablecimiento. El análisis de estado estable incluye (Grande-Moran \& Feltes, 2014):

- Análisis de control de voltaje y sobretensiones de estado estable después de la energización de las líneas de transmisión (Efecto Ferranti). El control de voltaje se aplica mediante la capacidad de potencia reactiva de las unidades de arranque negro, sistema de carga y, cuando esté disponible, compensación de derivación reactiva (inductores y capacitores) y taps bajo carga de transformadores. 
- Confirmación de la capacidad de las unidades de blackstarting para absorber la potencia reactiva producida por la capacidad de carga del sistema de transmisión.

- Simulación paso a paso del plan de blackstart que se está probando para garantizar su viabilidad y cumplimiento con límites operacionales requeridos.

- Verificación de la robustez del plan de blackstart probado para asegurar su capacidad para compensar la indisponibilidad de componentes clave para ser utilizados en el plan.

- Demostración de generación y capacidad de comparación de carga.

En la figura 4 se evalúan el tiempo optimista, el tiempo pesimista y un tiempo de rendimiento. El tiempo de rendimiento es el tiempo más probable, sin embargo, como la rapidez de la realización de las maniobras depende de los operarios y los sistemas de control, se considera cierta desviación para cada una. El tiempo de restablecimiento asciende a 6.57 horas, y los picos más altos en las gráficas corresponde a la energización de grandes plantas térmicas a vapor.

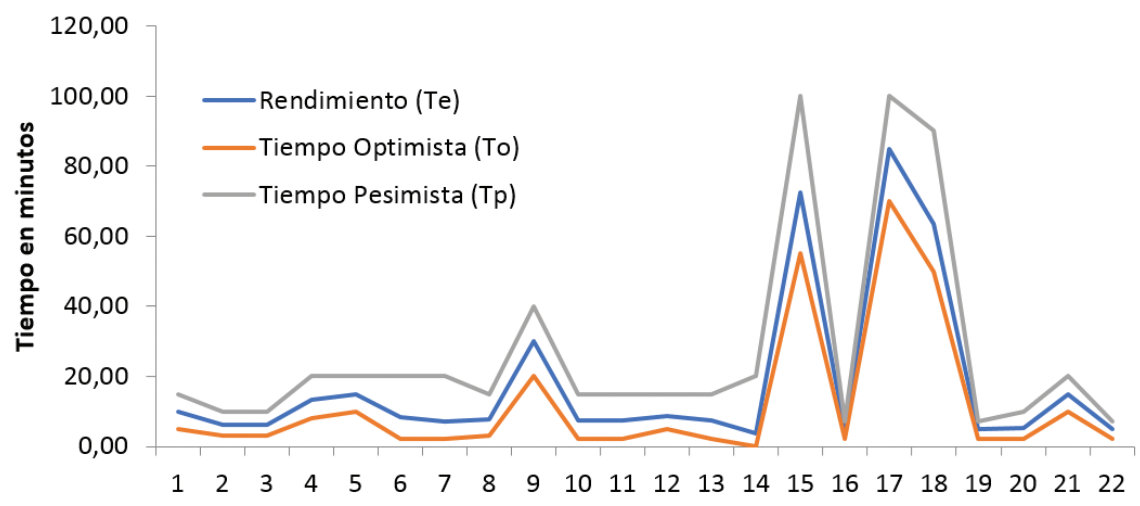

Figura 4. Evaluación del tiempo estimado de restablecimiento en la zona central

En la tabla 5 se estiman los tiempos que tardarían en ser realizadas las maniobras de acuerdo a la experiencia y operación del SENI en tiempo real (De León, 2007). Se contemplan a su vez, un tiempo de rendimiento y dos escenarios, uno más crítico y otro óptimo. 
En la figura 5 se muestran los perfiles de tensión en cada una de las subestaciones a considerar una vez realizadas todas las maniobras. Cabe destacar que el comportamiento de la tensión es dinámico y va variando cada vez que se añade un generador al sistema o se asume cierto valor de carga, pero el mismo nunca cae por debajo del valor por unidad.

Tabla 5. Maniobras de Restablecimiento en la Zona Central

\begin{tabular}{|c|c|c|c|c|c|c|c|}
\hline No & Descripción & $\begin{array}{l}\text { Tiempo } \\
\text { Optimista } \\
\text { (To) }\end{array}$ & $\begin{array}{l}\text { Tiempo Más } \\
\text { Probable } \\
\text { (TM) }\end{array}$ & $\begin{array}{l}\text { Tiempo } \\
\text { Pesimista } \\
\text { (Tp) }\end{array}$ & $\begin{array}{c}\text { Rendimiento } \\
\text { (Te) }\end{array}$ & $\begin{array}{l}\text { Desviación } \\
\text { Estándar } \\
(\sigma)\end{array}$ & $\begin{array}{l}\text { Varianza } \\
(\sigma)\end{array}$ \\
\hline 1 & $\begin{array}{c}\text { Arrancar Jiguey y entrar } \\
\text { en línea }\end{array}$ & 5 & 10 & 15 & 10 & 1.67 & 2.78 \\
\hline 2 & $\begin{array}{c}\text { Energizar S/E Pizarrete } \\
\text { y tomar carga }\end{array}$ & 3 & 6 & 10 & 6.17 & 1.17 & 1.36 \\
\hline 3 & $\begin{array}{c}\text { Energizar S/E Palamara } \\
\text { y tomar carga }\end{array}$ & 3 & 6 & 10 & 6.17 & 1.17 & 1.36 \\
\hline 4 & $\begin{array}{l}\text { Energizar Valdesia y } \\
\text { entregar potencia }\end{array}$ & 8 & 13 & 20 & 13.33 & 2 & 4 \\
\hline 5 & $\begin{array}{c}\text { Energizar los diesel de } \\
\text { Palamara y entregar } \\
\text { potencia }\end{array}$ & 10 & 15 & 20 & 15 & 1.67 & 2.78 \\
\hline 6 & $\begin{array}{l}\text { Energizar S/E Arroyo } \\
\text { Hondo y tomar carga }\end{array}$ & 2 & 7 & 20 & 8.33 & 3 & 9 \\
\hline 7 & $\begin{array}{l}\text { Energizar S/E La } 40 \text { y } \\
\text { tomar carga }\end{array}$ & 2 & 5 & 20 & 7 & 3 & 9 \\
\hline 8 & $\begin{array}{l}\text { Energizar S/E Timbeque } \\
\text { II y tomar carga }\end{array}$ & 3 & 7 & 15 & 7.67 & 2 & 4 \\
\hline 9 & $\begin{array}{l}\text { Energizar Estrella del } \\
\text { Mar (SEABOARD) y } \\
\text { entregar potencia }\end{array}$ & 20 & 30 & 40 & 30 & 3.33 & 11.11 \\
\hline 10 & $\begin{array}{l}\text { Energizar S/E CNP y } \\
\text { tomar carga }\end{array}$ & 2 & 7 & 15 & 7.5 & 2.17 & 4.69 \\
\hline 11 & $\begin{array}{l}\text { Energizar S/E Los Mina } \\
\text { y tomar carga }\end{array}$ & 2 & 7 & 15 & 7.5 & 2.17 & 4.69 \\
\hline 12 & $\begin{array}{c}\text { Energizar Los Mina } \\
\text { V \& VI y entregar } \\
\text { potencia }\end{array}$ & 5 & 8 & 15 & 8.67 & 1.67 & 2.78 \\
\hline 13 & $\begin{array}{c}\text { potencia } \\
\text { Energizar S/E } \\
\text { Hainamosa y tomar } \\
\text { carga }\end{array}$ & 2 & 7 & 15 & 7.5 & 2.17 & 4.69 \\
\hline 14 & $\begin{array}{c}\text { Energizar S/E } \\
\text { Interconexión Andrés }\end{array}$ & 0.15 & 0.5 & 20 & 3.69 & 3.31 & 10.95 \\
\hline 15 & $\begin{array}{c}\text { Energizar S/E Planta } \\
\text { AES Andrés y entregar } \\
\text { potencia }\end{array}$ & 55 & 70 & 100 & 72.5 & 7.5 & 56.25 \\
\hline 16 & $\begin{array}{c}\text { Energizar S/E SPM II y } \\
\text { tomar carga }\end{array}$ & 2 & 5 & 7 & 4.83 & 0.83 & 0.69 \\
\hline 17 & $\begin{array}{l}\text { Energizar Itabo y } \\
\text { entregar potencia }\end{array}$ & 70 & 85 & 100 & 85 & 5 & 25 \\
\hline 18 & $\begin{array}{l}\text { Energizar Haina y } \\
\text { entregar potencia }\end{array}$ & 50 & 60 & 90 & 63.33 & 6.67 & 44.44 \\
\hline 19 & $\begin{array}{c}\text { Energizar S/E Villa } \\
\text { Duarte y tomar carga }\end{array}$ & 2 & 5 & 7 & 4.83 & 0.83 & 0.69 \\
\hline 20 & $\begin{array}{c}\text { Energizar S/E km } 15 \\
\text { Azua y tomar carga }\end{array}$ & 2 & 5 & 10 & 5.33 & 1.33 & 1.78 \\
\hline
\end{tabular}




\begin{tabular}{|c|c|c|c|c|c|c|c|}
\hline No & Descripción & $\begin{array}{c}\text { Tiempo } \\
\text { Optimista } \\
(\mathbf{T o})\end{array}$ & $\begin{array}{c}\text { Tiempo Más } \\
\text { Probable } \\
(\mathbf{T M})\end{array}$ & $\begin{array}{c}\text { Tiempo } \\
\text { Pesimista } \\
(\mathbf{T} \mathbf{p})\end{array}$ & $\begin{array}{c}\text { Rendimiento } \\
(\mathbf{T e})\end{array}$ & $\begin{array}{c}\text { Desviación } \\
\text { Estándar } \\
(\boldsymbol{\sigma})\end{array}$ & $\begin{array}{c}\text { Varianza } \\
(\boldsymbol{\sigma})\end{array}$ \\
\hline 21 & $\begin{array}{c}\text { Energizar Monte Río y } \\
\text { entregar potencia }\end{array}$ & 10 & 15 & 20 & 15 & 1.67 & 2.78 \\
\hline 22 & $\begin{array}{c}\text { Energizar S/E Barahona } \\
\text { Carbón y tomar carga }\end{array}$ & 2 & 5 & 7 & 4.83 & 0.83 & 0.69 \\
\hline & TOTAL en minutos & $\mathbf{2 6 0 . 1 5}$ & $\mathbf{3 7 8 . 5}$ & $\mathbf{5 9 1}$ & $\mathbf{3 9 4 . 1 9}$ & & $\mathbf{9 . 3 4}$ \\
\hline & TOTAL en horas & $\mathbf{4 . 3 4}$ & $\mathbf{6 . 3 1}$ & $\mathbf{9 . 8 5}$ & $\mathbf{6 . 5 7}$ & & $\mathbf{0 . 1 6}$ \\
\hline
\end{tabular}

Perfiles de tensión en Nodos Zona Central

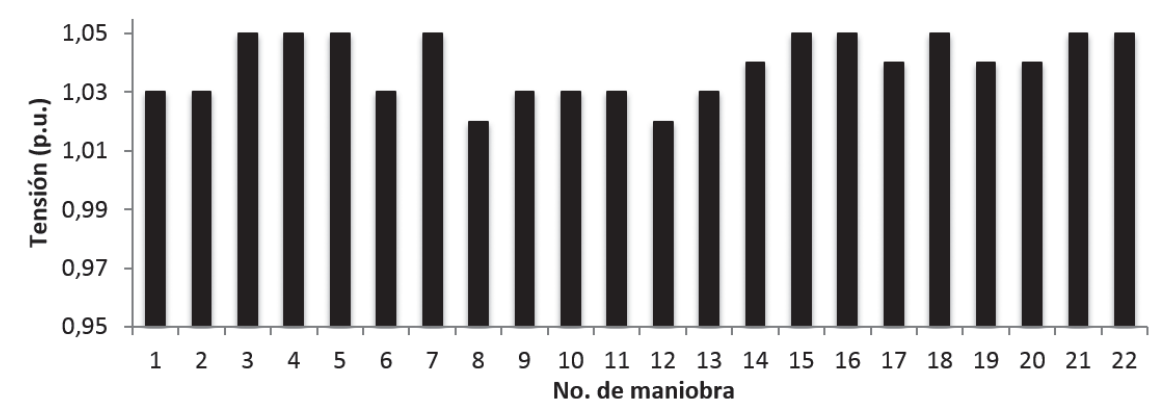

Figura 5. Diagrama esquemático de subestaciones para restablecimiento en la zona central

\section{Impacto de la propuesta a implementar}

\section{Estudio económico-social}

El ultimo blackout que sufrió el sistema eléctrico interconectado según el Centro de Control de Energía sucedió el 16 de mayo del 2015 y se extendió hasta el día siguiente, el mismo fue causado, como se ha mencionado, por el desprendimiento del aislador de una cuchilla y tuvo una duración de 24 horas. Para determinar las pérdidas macroeconómicas se utilizará la energía perdida durante el evento, la cual fue $\mathbf{2 2 , 3 8 5 . 3 7} \mathbf{M W h}$; $34.20 \%$ para el sector residencial, $36.60 \%$ para el sector industrial, $23 \%$ en el sector comercial y de servicios públicos, $5.80 \%$ en el sector transporte, agroindustrial y minero, y $0.40 \%$ en el sector de la construcción. Esto puede traducirse en pérdidas económicas totales estimadas de RD\$ 40,757,229.16 (Centro de Control de Energía, 2015). 
Con las simulaciones realizadas y los estudios de tiempo de restauración del sistema eléctrico mencionados, se realizaron estudios comparativos entre las pérdidas económicas antes las diferentes condiciones del sistema. En la tabla 6 se presenta un posible escenario de qué hubiese ocurrido si para el último blackout se hubiese puesto en vigencia la metodología planteada en este trabajo.

Tabla 6. Comparación del impacto de la metodología de re-establecimiento

\begin{tabular}{|c|c|c|c|c|c|c|}
\hline \multicolumn{7}{|c|}{$\begin{array}{l}\text { ARRANQUE BLACKSTART CON METODOLOGÍA DE RESTABLECIMIENTO VERSUS SIN METODOLOGÍA } \\
\text { DE RESTABLECIMIENTO }\end{array}$} \\
\hline $\begin{array}{c}\text { Valor total de las } \\
\text { estimadas pérdidas } \\
\text { con metodología } \\
(\mathrm{RD} \$)\end{array}$ & $\begin{array}{l}\text { Residencial } \\
\text { (RD\$) }\end{array}$ & $\begin{array}{l}\text { Industrial } \\
(\mathrm{RD} \$)\end{array}$ & $\begin{array}{l}\text { Comercial } \\
\text { y servicio } \\
\text { público } \\
\text { (RD\$) }\end{array}$ & $\begin{array}{c}\text { Transporte, } \\
\text { agroindustrial } \\
\text { y minero } \\
\text { (RD\$) }\end{array}$ & $\begin{array}{l}\text { Construcción } \\
\quad(\mathrm{RD} \$)\end{array}$ & TOTAL \\
\hline Porcentaje & $34 \%$ & $37 \%$ & $23 \%$ & $6 \%$ & $0 \%$ & $100 \%$ \\
\hline $\begin{array}{c}\text { SIN } \\
\text { METODOLOGÍA }\end{array}$ & $13,938,972.39$ & $14,917,145.89$ & $9,374,162.72$ & $2,363,919.23$ & $163,028.92$ & $40,757,229.16$ \\
\hline \multirow[t]{2}{*}{$\begin{array}{c}\text { CON } \\
\text { METODOLOGÍA }\end{array}$} & $3,815,793.69$ & $4,083,568.69$ & $2,566,177.04$ & $647,122.91$ & $44,629.17$ & $11,157,291.50$ \\
\hline & & & & & Ahorro RD \$ & $29,599,937.66$ \\
\hline
\end{tabular}

Con esto se puede ver el monto ahorrado de RD\$29,599,937.66 en la economía del país, si este tuviese en disposición una metodología de restablecimiento. Este monto representa el 2.09 \% para el año 2015 del Fondo de Estabilización de la Tarifa Eléctrica (FETE).

\section{Conclusiones}

Tras la realización de este trabajo se puede concluir que el establecimiento de una metodología ante blackouts es de vital importancia para el sistema eléctrico dominicano, ya que, como se evidenció anteriormente, mientras más prolongados son los blackouts mayores son las pérdidas asociadas, provocando así que se agudice la crisis financiera que caracteriza el sector eléctrico. Ejemplo claro de esto es que el valor de las pérdidas estimadas en el último blackout alcanzó alrededor del $2.88 \%$ del fondo de estabilización de la tarifa eléctrica. En este también se analizaron una serie de metodologías de remuneración para ver cuál se adaptaba mejor 
al sistema eléctrico dominicano, siendo esta el contrato bilateral, puesto que este es un concreto sistema de pago con condiciones claras y justas que a la vez hace que sus agentes maximicen sus ganancias económicas por su flexibilidad.

Este documento describe las operaciones de restauración y los estudios que deben formar parte de un proceso de planificación de restauración. En particular, intentó abordar muchos de los problemas técnicos, incluida la dinámica del sistema y los aspectos de control del proceso de blackstart. El desarrollo de planes de restauración completos y la prueba de dichos planes mediante simulación y simulacros ayudarán a minimizar la interrupción del servicio a cargas críticas y el riesgo de daños al equipo luego de apagones parciales o totales del sistema de energía.

\section{Agradecimientos}

A la gerencia de operación de Organismo Coordinador del Sistema Eléctrico Nacional Interconectado (OC-SENI) por el acompańamiento en las simulaciones y escenarios en el software DIgSILENT, a la gerencia de planificación Comercial de la Corporación Dominicana de Empresas eléctricas Estatales (CDEEE) por sus valiosas indicaciones y recomendaciones, además de la gerencia de compra de energía de la Empresa Distribuidora de Electricidad del Este (EDE Este) por su colaboración en la documentación de la propuesta remunerativa propuesta.

\section{Referencias}

Abedi, A., Gaudard, L., \& Romerio, F. (2019). Review of major approaches to analyze vulnerability in power system. Reliability Engineering \& System Safety, 183, 153-172. doi: https://doi.org/10.1016/j. ress.2018.11.019

Centro de Control de Energía, (2018). Histórico de Blackout. In E. d. t. e. Dominicana (Ed.).

Grande-Moran, C., \& Feltes, J. W. J. S. P. T. I., Inc. (2014). An Overview of Restoration Issues and Blackstart Analysis. 
De León, E. (2007). Aplicación de un criterio de restauración al sistema eléctrico dominicano (tesis de maestría). Recinto Universitario de Mayagüez, Puerto Rico.

Hou, Y., Liu, C.-C., Zhang, P., \& Sun, K. (2009). Constructing power system restoration strategies. Paper presented at the 2009 International Conference on Electrical and Electronics Engineering-ELECO 2009.

Janda, K. (2018). Slovak electricity market and the price merit order effect of photovoltaics. Energy Policy, 122, 551-562. doi: https:// doi.org/10.1016/j.enpol.2018.07.021

Kamali, S., \& Amraee, T. (2017). Blackout prediction in interconnected electric energy systems considering generation re-dispatch and energy curtailment. Applied Energy, 187, 50-61. doi: https:// doi.org/10.1016/j.apenergy. 2016.11.040

Kurup, S. R., \& Ashok, S. (2015, 19-21 Nov. 2015). Grid restoration planning after a total blackout. Paper presented at the 2015 International Conference on Control Communication \& Computing India (ICCC).

Leng, Y., Lu, Q., \& Liang, C. (2018). Black-start decision making based on collaborative filtering for power system restoration. International Journal of Electrical Power \& Energy Systems, 100, 279-286. doi: https://doi.org/10.1016/j.ijepes.2018.02.044

Masnicki, R., \& Mindykowski, J. (2017). Coordination of operations in registration channel of data from electrical power system. Measurement, 99, 68-77. doi: https://doi.org/10.1016/j.measurement.2016.12.013

Mota, A. A., Mota, L. T. M., \& Morelato, A. (2007). Visualization of Power System Restoration Plans Using CPM/PERT Graphs. IEEE Transactions on Power Systems, 22(3), 1322-1329. doi:10.1109/ TPWRS.2007.901118

Organismo Coordinador del Sistema Eléctrico Nacional Interconectado, O. C. d. S. E. N. I. (2015). Informe diario de operación. In (pp. 3)

Organismo Coordinador del Sistema Eléctrico Nacional Interconectado, O. C. d. S. E. N. I. (2018). Informe Anual 2018 del SENI 110.

Wu, Y.-K., Chang, S. M., \& Hu, Y.-L. (2017). Literature Review of 
Power System Blackouts. Energy Procedia, 141, 428-431. doi: https:// doi.org/10.1016/j.egypro.2017.11.055

Yang, J., Liu, W., \& Liu, P. (2014). Application of SMES Unit in Black Start. Physics Procedia, 58, 277-281. doi: https://doi.org/10.1016/j. phpro.2014.09.050

Yusuf, J., Sakib, F., \& Ahsan, Q. (2016, 20-22 Dec. 2016). Study of blackout prevention of a power system. Paper presented at the 2016 9th International Conference on Electrical and Computer Engineering (ICECE). 